\title{
Ocular Pharmacokinetics of Fluocinolone Acetonide Following Iluvien Implantation in the Vitreous Humor of Rabbits
}

\author{
Frances E. Kane ${ }^{1}$ and Kenneth E. Green ${ }^{2}$
}

\begin{abstract}
Purpose: The purpose of this study was to evaluate the systemic and ocular pharmacokinetics (PK) of fluocinolone acetonide (FAc) following administration of Iluvien ${ }^{\circledR}$ intravitreal implants.

Methods: The FAc intravitreal implant was administered to rabbits in 3 doses $(0.2,0.5$, and $1.0 \mu \mathrm{g} / \mathrm{day})$. The concentration of FAc was measured by a validated liquid chromatography-mass spectrometry/mass spectrometry (LC-MS/MS) method in plasma and ocular tissues at various time points through month 24.

Results: Following administration of the $0.2 \mu \mathrm{g} /$ day implant, FAc levels peaked in most tissues at day 2 or 8 , reached approximate steady state levels by month 3 and very gradually decreased over the duration of the study. The FAc level in the aqueous humor was not measurable at most time points in the rabbit. FAc was still present in most ocular tissues at 2 years. The 0.5 and $1.0 \mu \mathrm{g} /$ day dose groups followed the same pattern through month 9. The elimination half lives in the tissues for which it was measurable were greater than 83 days. Exposure to FAc was highest in the choroid/retinal pigment epithelium for all doses, followed by lens and retina.

Conclusions: The results of this study demonstrate sustained delivery of FAc from the Iluvien intravitreal implant in the ocular tissue of rabbits. Retina and lens FAc levels with the Iluvien implant were approximately $1 / 10$ those reported with the Retisert ${ }^{\circledR}$ implant. FAc levels in the aqueous were not measureable with Iluvien where they were measured for 12 months with Retisert.
\end{abstract}

\section{Introduction}

C HRONIC OCULAR DISEASES such as diabetic macular edema and uveitis may require long-term treatment with corticosteroid; however, systemic corticosteroid treatment is associated with significant side effects and repeated intravitreal injections are associated with the risk of endophthalmitis, retinal detachment, iritis/uveitis, ocular hypertension, cataract, and intraocular hemorrhage. ${ }^{1}$ Local delivery of the lowest therapeutic corticosteroid dose over long periods is the best therapeutic strategy to avoid adverse events due to the therapeutic agent and the administration procedure. Thus, the need for long-term corticosteroid treatment of chronic ocular diseases has led to the development of sustained-release intravitreal products.

Retisert $^{\circledR}$ (fluocinolone acetonide intravitreal implant) was the first sustained-release intravitreal product approved for noninfectious uveitis of the posterior segment of the eye. This nonbioerodable surgical implant releases fluocinolone acetonide $(\mathrm{FAc})$ at a nominal initial rate of $0.6 \mu \mathrm{g} / \mathrm{day}$ with steady state release of $0.3-0.4 \mu \mathrm{g} / \mathrm{day}$ for $\sim 30$ months. $^{2}$ Following the development of Retisert, Control Delivery Systems (now pSivida, Inc., Watertown, MA) created an injectable implant to allow administration into the vitreous through a 25 -gauge needle. The implant is preloaded into a handheld device optimized for the intravitreal delivery of the implant through the pars plana. The new implant, Iluvien ${ }^{\circledR}(190 \mu \mathrm{g}$ FAc intravitreal implant in applicator; Alimera Sciences, Inc., Alpharetta, GA), has an initial release rate of $0.23 \mu \mathrm{g} / \mathrm{day}$ FAc and steady state release levels of $\sim 0.1 \mu \mathrm{g} / \mathrm{day} .{ }^{3}$ The product has been approved in Europe for the treatment of vision impairment associated with chronic diabetic macular edema considered insufficiently responsive to available therapies and is also approved in the United States on September 26, 2014.

This study examines the ocular pharmacokinetics (PK) of FAc following intravitreal administration of Iluvien in rabbits over a 2-year period and demonstrates that FAc is delivered at consistent low levels to ocular tissues. The results are compared to published data for a similar study of the Retisert implant by Driot et al. ${ }^{4}$

\footnotetext{
${ }^{1}$ Apropos Pharmaceutical Consulting LLC, Suwanee, Georgia.

${ }^{2}$ Alimera Sciences, Inc., Alpharetta, Georgia.
} 


\section{Methods}

This study was performed in accordance with Good Laboratory Practices at Charles River Laboratories (Redfield, AR) in support of a long-term ocular toxicity study. The study was conducted in accordance with the Association for Research in Vision and Ophthalmology statement for the Use of Animals in Ophthalmic and Vision Research.

Dutch Belted Rabbits (5-6 months old) were received from Myrtle's Rabbitry (Thompson Station, TN) and allowed to acclimatize for 3-7 days. Male rabbits weighing $2.2-2.6 \mathrm{~kg}$ and females weighing $2.0-2.5 \mathrm{~kg}$ were individually housed in stainless steel cages and provided 100-125 g rabbit chow (Purina Certified Rabbit Diet PMI 5325) daily and filtered water ad libitum.

The FAc implant $(0.2$ or $0.5 \mu \mathrm{g} /$ day $)$ was administered via intravitreal injection to both eyes of 5-6-month-old male and female rabbits using aseptic technique and topical anesthesia (Table 1). For group 1, the $0.2 \mu \mathrm{g}$ /day implant was administered once on study day 1 . Group 2 received the $0.5 \mu \mathrm{g} /$ day implant on study day 1 and during week 52. For group 3, two $0.5 \mu \mathrm{g} /$ day implants were administered on study day 1 , and during week 52 using injectors containing 2 FAc implants (Table 1). Following the dosing procedure an ophthalmic lubricant (Puralube Ophthalmic Ointment) was applied to both eyes of each animal to prevent dryness and irritation. In rabbits dosed in parallel to the pharmacokinetic animals, there were no signs of ocular toxicity, which could impact on the release, distribution, or elimination of FAc from the eye.

Blood samples were collected into tubes containing $\mathrm{K}_{3}$ EDTA on day $2(24 \mathrm{~h} \pm 30 \mathrm{~min}$ postdose $)$, day 8 , and weeks $5,8,13,26,39,52$ ( $24 \mathrm{~h}$ postdose), 78, and 104. The blood samples were centrifuged and the plasma was extracted. Aqueous humor $(\sim 100 \mu \mathrm{L})$ was aspirated from the anterior chamber using a 25-gauge or smaller needle immediately after euthanasia. The globe was removed by transconjunctival enucleation and dissection along the sclera and optic nerve. An initial shallow incision was made posterior to the limbus with a scalpel and circumferentially extended using corneoscleral scissors. The FAc implant was removed using forceps, stored separately, and the vitreous was removed. The lens was removed from the anterior cap using smooth forceps or spatula. The cornea was separated from the iris using corneoscleral scissors. (The method for analysis of the cornea was not sufficiently consistent to pass acceptance standards so data for this tissue are not included.) Using fine forceps, the iris and ciliary body (CB) were pulled from the sclera. The remaining eye cup was cut at

Table 1. Intravitreal Administration Plan FOR FAC IMPLANTS

\begin{tabular}{|c|c|c|c|c|}
\hline \multirow{2}{*}{$\begin{array}{l}\text { Group } \\
\text { number }\end{array}$} & \multicolumn{2}{|c|}{ Number of rabbits } & \multirow{2}{*}{$\begin{array}{c}\text { Number of } \\
\text { implants }\end{array}$} & \multirow{2}{*}{$\begin{array}{c}\text { Nominal daily } \\
\text { dose of FAc } \\
(\mu g / \text { day })\end{array}$} \\
\hline & Males & Females & & \\
\hline 1 & 10 & 10 & 1 & 0.2 \\
\hline 2 & 10 & 10 & $2^{\mathrm{a}}$ & 0.5 \\
\hline 3 & 10 & 10 & $4^{b}$ & 1.0 \\
\hline
\end{tabular}

${ }^{\mathrm{a} O}$ One device on study day 1 , and another during week 52 .

${ }^{\mathrm{b}}$ Two devices on study day 1 , and 2 during week 52 .

FAc, fluocinolone acetonide. several locations to allow access to the retina. Using a dissection microscope the retina was peeled away from the retinal pigment epithelium (RPE). (Cross-contamination was minimized to the extent possible; however, due to the delicate nature of the retina, some cross-contamination between retina and vitreous cannot be ruled out.) The choroid (C) and RPE were peeled off the sclera with smooth forceps. Each sample was placed in a $600 \mu \mathrm{L}$ vial on dry ice and stored frozen at $-70^{\circ} \mathrm{C}$ until shipment on dry ice to the analytical laboratory. All samples were stored in separate containers, labeled, placed on dry ice, and stored at approximately $-70^{\circ} \mathrm{C}$ until shipment to Huntingdon Life Sciences, Inc. (Somerset, NJ).

The concentrations of FAc in rabbit plasma and ocular tissues were measured using a validated liquid chromatography-mass spectrometry/mass spectrometry (LC-MS/MS) method at Huntingdon Life Sciences, Inc. Dexamethasone 21-acetate (Sigma-Aldrich, St. Louis, MO) or triamcinolone-6-d1 acetonide-d6 (CDN Isotopes, Inc., Pointe-Claire, Quebec, Canada) were used as internal standards. The internal standard used for the initial validation of this method (several years before this study) was dexamethasone 21acetate. When the analytical method was requalified for use in this study, newer LC-MS/MS equipment was used and the method was requalified; over time some variability in tracking FAc was observed, so triamcinolone-6-d1 acetonide-d6 was tested. This deuterated compound provided a more consistent internal standard and the method was partially revalidated using the new internal standard.

The method of extraction was based on liquid-liquid procedure with a mixture of chlorobutane and chloroform (90:10) as an extraction solvent. The organic layer was evaporated to dryness, reconstituted with mobile phase, and directly injected into an LC-MS/MS instrument for detection and quantification. Separation of analytes was accomplished with an ODS-A C18 column (100 mm length, $3.0 \mathrm{~mm}$ diameter, $3 \mu \mathrm{m}$ particle size; YMC America, Inc., Allentown, PA) with a mobile phase of acetonitrile $(55 \%) /$ $10 \mathrm{mN}$ ammonium acetate $(40 \%) /$ methanol $(5 \%)$ at a flow rate of $0.6 \mathrm{~mL} / \mathrm{min}$. An enhanced quadripole mass spectrometer (PE Sciex API 3000) with heated nebulizer ion source in positive ion mode detecting ion pairs for FAc (453.3/413.0 amu), dexamethasone 21-acetate (435.5/ $397.0 \mathrm{amu}$ ), and triamcinolone-6-d1 acetonide-d6 (442.4/ $404.3 \mathrm{amu}$ ) was used for quantification. Samples were analyzed in batches throughout the study. For each batch of samples a calibration curve was constructed from duplicate measurements of 10 concentrations of FAc in rabbit plasma over the concentration range of $200-51,200 \mathrm{pg} / \mathrm{mL}$, using $1 / \mathrm{x}$ weighted linear regression analysis. Data collection and peak area integration were performed using PC Analyst version 1.2 or 1.4.2 software in conjunction with the mass spectrometer. Subsequent data processing and quantification was performed using Watson LIMS V6.1.1.04 (ThermoFisher Scientific, Waltham, MA). The lower limit of quantification was $200 \mathrm{pg} / \mathrm{mL}$.

The validation of the bioanalytical method included establishing standard procedures, defining acceptance procedures, demonstrating an absence of interference at the retention time of FAc or the internal standards, and demonstration of linearity of the function of $1 /$ concentration over the range defined with a correlation coefficient of 0.9991 of better. The inter-assay coefficient of variation for 
Table 2. Summary of Mean FAc Concentrations in Ocular Tissues of RabBits Treated WITH FAC IMPLANT

\begin{tabular}{lccc}
\hline & \multicolumn{3}{c}{ FAc concentration $(\mathrm{ng} / \mathrm{g})$} \\
\cline { 2 - 4 } Study day & $\begin{array}{c}0.2 \mu \mathrm{g} / \text { day } \\
\text { implant }\end{array}$ & $\begin{array}{c}\text { 0.5 } \mathrm{gg} / \text { day } \\
\text { implant }\end{array}$ & $\begin{array}{c}1.0 \mu \mathrm{g} / \mathrm{day} \\
\text { implants } \\
\text { implay }\end{array}$ \\
\hline
\end{tabular}

\begin{tabular}{ll}
\hline Aqueous humor & \\
2 & 0.0515 \\
8 & 0 \\
29 & 0.0985 \\
55 & 0 \\
89 & 0 \\
182 & 0 \\
271 & 0 \\
363 & 0 \\
539 & 0 \\
728 & 0
\end{tabular}

Vitreous humor 29

Lens

\section{Retina}

Choroid/PE

\subsection{6}

0.988

0.507

1.02

0.261

0.367

0.127

0.176

0.0947

0.0398

\subsection{1}

3.45

5.03

18.8

2.32

2.59

2.51

2.78

3.55

1.33

\section{2}

8.66

5.06

8.71

0

4.80

0

0

0

0

63.9

42.2

35.4

22.8

9.45

12.2

8.14

5.93

2.80

3.15
0

0.190

0.188

0

0

0

0

0

0

0

35.9

18.5

5.40

4.39

2.65

1.70

1.26

13.6

1.56

1.23

27.9

154

25.2

52.5

23.3

12.7

11.2

604

48.4

58.9

142

125

22.6

30.3

13.5

0

5.75

69.3

38.2

6.18

12.0

0

136

169

173

124

67.6

57.5

37.7

161

87.2

23.5

TABle 2. (CONTINUED)

\begin{tabular}{|c|c|c|c|}
\hline \multirow[b]{2}{*}{ Study day } & \multicolumn{3}{|c|}{ FAc concentration $(n g / g)$} \\
\hline & $\begin{array}{c}0.2 \mu \mathrm{g} / \mathrm{day} \\
\text { implant }\end{array}$ & $\begin{array}{c}0.5 \mu \mathrm{g} / \text { day } \\
\text { implant }\end{array}$ & $\begin{array}{c}1.0 \mu \mathrm{g} / \mathrm{day} \\
2-0.5 \mu \mathrm{g} / \mathrm{day} \\
\text { implants }\end{array}$ \\
\hline \multicolumn{4}{|c|}{ Iris/ciliary body } \\
\hline 2 & 24.2 & 36.2 & 81.5 \\
\hline 8 & 27.1 & 17.0 & 114 \\
\hline 29 & 7.83 & 28.0 & 78.4 \\
\hline 55 & 20.6 & 11.9 & 49.0 \\
\hline 89 & 3.05 & 10.3 & 15.9 \\
\hline 182 & 15.5 & 14.7 & 27.5 \\
\hline 271 & 4.95 & 4.02 & 5.95 \\
\hline 363 & 3.05 & 26.3 & 27.2 \\
\hline 539 & 1.73 & 6.95 & 16.3 \\
\hline 728 & 0.563 & 3.31 & 8.50 \\
\hline
\end{tabular}

PE, pigment epithelium.

4 runs at the lower limit of quantification $(200 \mathrm{pg} / \mathrm{mL}$ was determined to be $9.9 \%$ with a relative error of $10.5 \%$ for FAc in rabbit plasma.

Pharmacokinetic analysis of the plasma and tissue concentration data was performed by Charles River Laboratories Preclinical Services (Montreal, Canada) based on noncompartmental modeling of the tissue concentrations for each group. Concentration values reported as not detectable or quantifiable were assigned a value of zero. The area under the concentration versus time curve (AUC) was calculated using the linear trapezoidal method if there were at least 3 quantifiable FAc concentrations at different time points. Where the coefficient of determination $\left(R^{2}\right)>0.8$ and sufficient data was available, the terminal elimination phase was identified based on the line of best fit using the final 3 observed concentration values.

\section{Results}

All plasma samples were below the limit of quantification $(200 \mathrm{pg} / \mathrm{mL})$. As there was no quantifiable systemic exposure to FAc following intravitreal administration of the FAc implant at $0.2,0.5$, or $1.0 \mu \mathrm{g} / \mathrm{day}$ doses, no pharmacokinetic analysis was performed.

The levels of FAc in the ocular tissues are provided in Table 2. Following administration of the $0.2 \mu \mathrm{g} / \mathrm{day}$ implant, FAc levels peaked in most tissues at day 2 or 8 , reached approximate steady state levels by month 3 , and very gradually decreased over the duration of the study. The FAc levels in tissues of chief relevance to diseases of the posterior pole (vitreous, retina, C/RPE) are graphically provided in Figure 1, along with aqueous humor results where measurable. FAc was still present in the vitreous, C/RPE, lens, and iris/ciliary body (I/CB) at 2 years, the final time point tested. The FAc level in the aqueous was below the lower limit of quantification $(200 \mathrm{pg} / \mathrm{mL})$ at most time points.

During the first 9 months, the 0.5 and $1.0 \mu \mathrm{g} /$ day implants followed the same pattern, with the maximum level of FAc at day 2 or 8 and gradual decrease in all tissues thereafter. The second administration of implants in these groups ( 1 or 2 additional $0.5 \mu \mathrm{g} /$ day implants, $\sim 3$ days before day 363 samples) increased FAc to levels similar to those seen with the initial implants and steady state ocular tissue concentrations of FAc were maintained following the second 

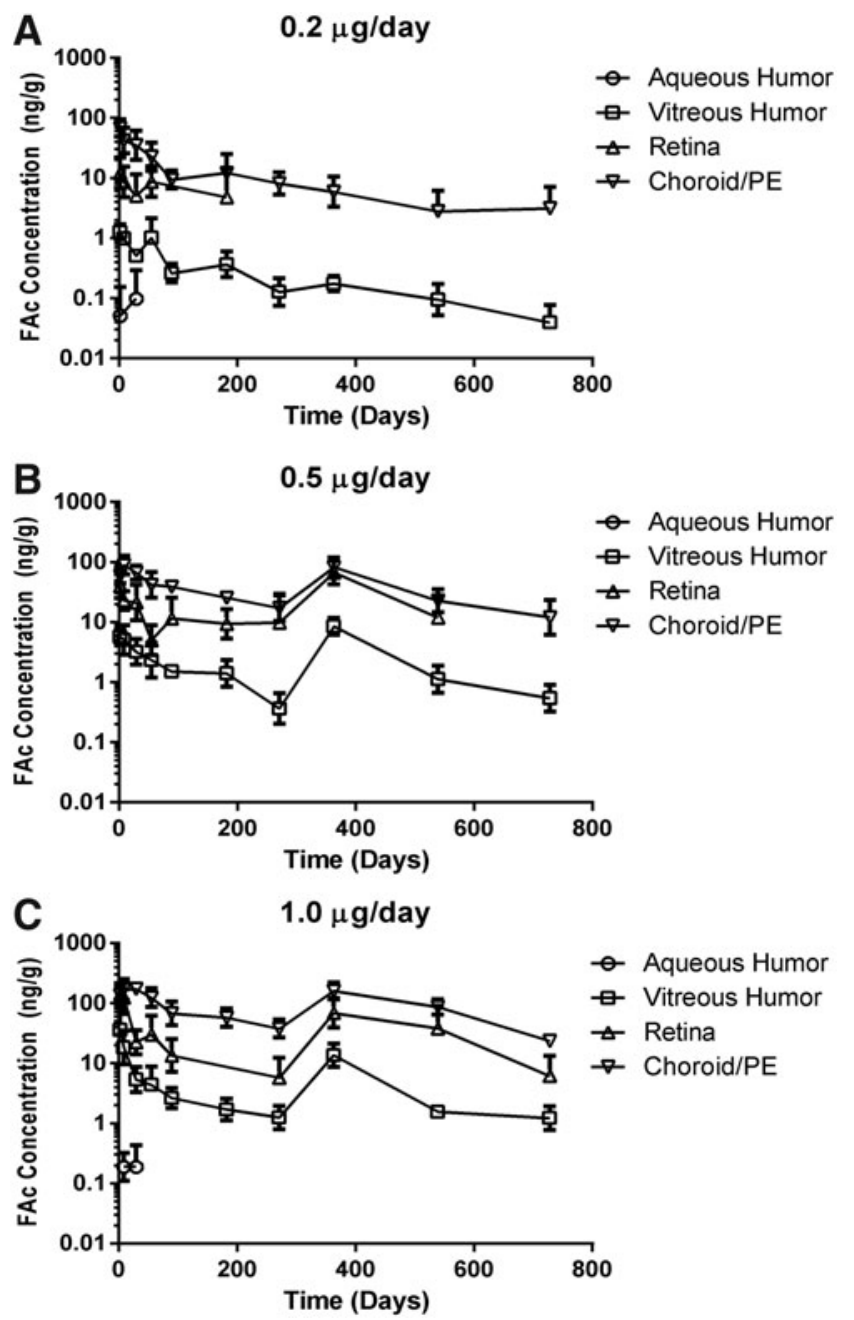

FIG. 1. Fluocinolone acetonide (FAc) concentrations in ocular tissues following intravitreal administration of the FAc implant. Data reported in each panel represent the tissue concentrations $(\mathrm{ng} / \mathrm{g}) \pm$ standard error of the mean for both eyes of 1 male and 1 female at each time point. (A) The levels of FAc in the vitreous humor and choroid/pigment epithelium were measurable for 2 years following the administration of one $0.2 \mu \mathrm{g} /$ day implant. (B) The levels of FAc in the retina, vitreous humor, and choroid/pigment epithelium were measurable through month 9 , when a second $0.5 \mu \mathrm{g} /$ day implant was administered. (C) When $1.0 \mu \mathrm{g} /$ day (two $0.5 \mu \mathrm{g} /$ day implants) is administered on day 1 , levels of FAc in the retina, vitreous humor, and choroid/pigment epithelium are approximately twice those observed with one $0.5 \mu \mathrm{g} /$ day implant through month 9. When 2 additional $0.5 \mu \mathrm{g}$ /day implants were administered at month 12, the FAc levels were similar to those after the first administration indicating that there was little accumulation. PE, pigment epithelium.

intravitreal injection of the implants. The levels of FAc remaining in all tissues 1 year after the second administration were essentially the same as those at week 52, indicating that the drug did not significantly accumulate in the eye.

Pharmacokinetic analyses determine the elimination halflife $\left(\mathrm{T}_{1 / 2}\right)$ and the area under the concentration versus time curve for FAc in each ocular tissue (Table 3). In most tissues, $\mathrm{T}_{1 / 2}$ exceeded $2,000 \mathrm{~h}$ (83 days) and did not appear to be dose or tissue dependent. The pharmacokinetic profile is consistent with concentration-limited sustained-release of FAc. In the vitreous the $\mathrm{T}_{1 / 2}$ was $2,305 \mathrm{~h}$ (96 days) for the dose which is marketed $(0.2 \mu \mathrm{g} /$ day $)$ for diabetic macular edema. Based on the $\mathrm{AUC}_{(0-\text { tlast })}(\mathrm{ng} * \mathrm{~h} / \mathrm{g})$ exposure to $\mathrm{FAc}$ was highest in the C/RPE for all doses, followed by lens and retina (Fig. 2).

\section{Discussion}

The results of this study demonstrate very long sustained delivery of FAc from the Iluvien intravitreal implant in the ocular tissue of rabbits. For each dose group, the highest level of FAc was observed in the C/RPE, followed by retina or iris $\mathrm{CB}$ and lens. The lowest levels of FAc were observed in the vitreous and aqueous humor. The presence of relatively high levels in the C/RPE and retina indicates that the implant efficiently delivers drug to the target tissues for posterior retinal diseases. The vitreous humor is a jelly-like substance containing very few cells or structural elements. As a result the levels in the vitreous are likely to be limited by the solubility of FAc (a hydrophobic steroid) in a primarily hydrophilic medium. The tissue distribution of FAc indicates that the drug diffuses through the vitreous and is taken up by the retina and other ocular tissues.

The lack of measurable FAc in the aqueous humor at most time points probably reflects lower diffusion of the drug into the anterior chamber in rabbits. This is contrast to the findings in humans wherein FAc is measurable through at least month 36 following administration of the implant. ${ }^{5}$ Interestingly, there was no quantifiable systemic exposure to FAc following intravitreal administration of the FAc implant at $0.2,0.5$, or $1.0 \mu \mathrm{g} /$ day doses. The animals in group 3 received 2 inserts in both eyes at day 1 and week 52. Assuming that the initial implants were releasing at approximately half the nominal rate and the newly administered implants were releasing at the nominal rate, $3 \mu \mathrm{g}$ FAc may have been released in each eye at the time the week 52 samples were taken. Despite this, FAc was not measurable in the systemic circulation, indicating efficient localization of the drug in the eye and rapid elimination of drug in the systemic circulation. This finding is consistent with the published half-life of FAc $(1.3-1.7 \mathrm{~h})$ due to hepatic metabolism and excretion by the kidneys (www.drugbank.ca).

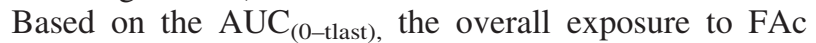
over 24 months follows essentially the same pattern as the concentration distribution by tissue; however, there was somewhat greater exposure over time in the lens than the retina with the 0.5 and $1.0 \mu \mathrm{g} /$ day doses. This may in part be due to differences in vascularity as the lens is avascular, while the retina is well-perfused.

The FAc intravitreal implant utilizes the same matrix permeated with FAc as was employed in Retisert, however, the injectable administration of Iluvien versus the surgical suturing of Retisert to the pars plana, results in a more posterior point of release. This more posterior point of release of Iluvien is combined with a daily release rate that is approximately one-half that exhibited by Retisert. Retisert contains $0.59 \mathrm{mg}$ of FAc (vs. $0.19 \mathrm{mg}$ for Iluvien) and is reported to achieve steady state levels of $0.3-0.4 \mu \mathrm{g} /$ day. ${ }^{2}$ Driot et al. described the pharmacokinetics of FAc release from 2 doses of Retisert implants (0.59 and $2.0 \mathrm{mg}$ ) 
Table 3. Pharmacokinetic Parameters for FAc in Ocular Tissues Following Intravitreal INJECTION OF FAC IMPLANTS

\begin{tabular}{llccc}
\hline Dose level $(\mu g /$ day) & \multicolumn{1}{c}{ Ocular tissues } & $T_{1 / 2}(h)$ & $C_{\max }(\mathrm{ng} / \mathrm{g})$ & $A U C_{(0-\text { tlast })}(\mathrm{ng} * \mathrm{~h} / \mathrm{g})$ \\
\hline 0.2 & Aqueous humor & $\mathrm{a}$ & 0.197 & \\
& Vitreous humor & 4,404 & 1.35 & 4,008 \\
& Lens & 6,284 & 19.2 & 60,092 \\
& Retina & $\mathrm{b}$ & 12.2 & 22,673 \\
& Choroid/pigment epithelium & $\mathrm{b}$ & 63.9 & 153,323 \\
& lris/ciliary body & 4,003 & 30.6 & 98,238 \\
0.5 & Aqueous humor & 2,305 & $\mathrm{a}$ & $\mathrm{a}$ \\
& Vitreous humor & $\mathrm{b}$ & 5.51 & 45,446 \\
& Lens & $\mathrm{a}$ & 67.4 & 440,154 \\
& Retina & 3,201 & 89.3 & 319,319 \\
& Choroid/pigment epithelium & 3,153 & 43.9 & 646,481 \\
& lris/ciliary body & 1 & 0.377 & 212,378 \\
& Aqueous humor & 3,056 & 43.4 & 79,638 \\
& Vitreous humor & $\mathrm{b}$ & 154 & 731,686 \\
& Lens & 2,434 & 173 & 546,891 \\
& Retina & 3,307 & 190 & $1,517,508$ \\
& Choroid/pigment epithelium & 5,230 & 114 & 395,429 \\
\hline
\end{tabular}

${ }^{a}$ PK parameters not estimated due to samples being BLQ or the terminal phase could not be identified.

${ }^{b}$ Values not reported because the AUC was extrapolated by more than $20 \%$ or $R^{2}$ was $<0.8$.

PK, pharmacokinetic.

in rabbits over 12 months. ${ }^{4}$ This study utilized the same rabbit species, analytical method, and analytical laboratory as the current study. The levels in C/RPE and I/CB with the Retisert implant were approximately twice those observed with the Iluvien implant. Retina and lens FAc levels were $\sim 10$-fold with the Retisert implant relative to the Iluvien implant, while the levels in the vitreous humor were 40 times higher. For the aqueous humor, only extremely low levels of FAc were detected at the earliest time points in this study, whereas they were detected over the entire 12 months of study with Retisert. These

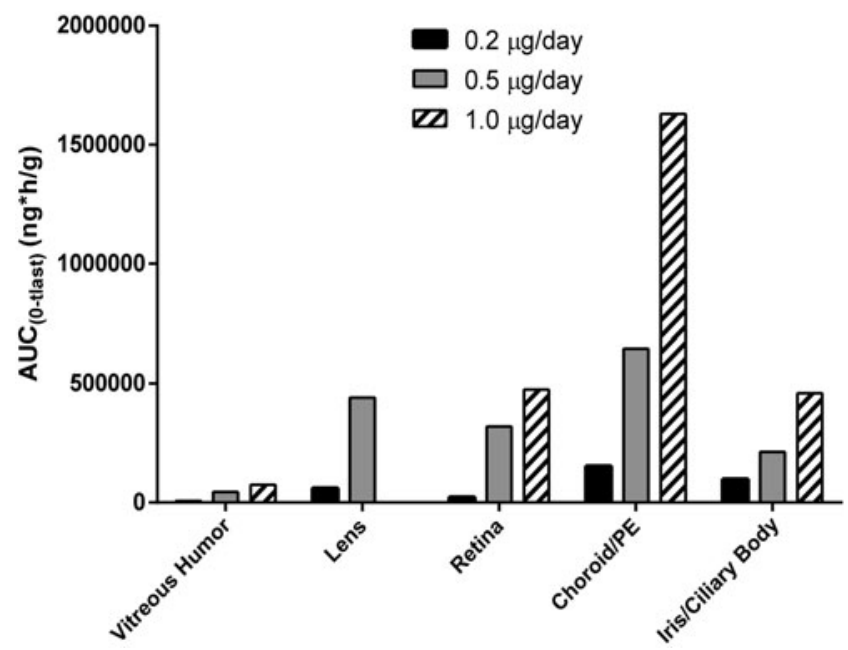

FIG. 2. $\mathrm{AUC}_{(0-\text { tlast })}$ in ocular tissues following intravitreal administration of the FAc implant. Based on the $\mathrm{AUC}_{(0-\text { tlast }}$ $\left(\mathrm{ng}^{*} \mathrm{~h} / \mathrm{g}\right)$ exposure to FAc increased by dose as expected. The value for the low dose group is disproportionately smaller because this group received a single intravitreal implant on day 1 while the 0.5 and $1.0 \mu \mathrm{g} /$ day groups received a second dose at month 12 . findings are consistent with the findings of Campochiaro et al., which demonstrated that FAc levels are higher in the aqueous humor of human subjects treated with Retisert than those treated with the FAc implant through 15 months (the last time point tested). ${ }^{5}$ This may also account for the higher rate of side effects of cataract and elevated intraocular pressure reported with Retisert compared with the FAc implant. $^{2,3}$

Limitations of this study include the sensitivity of the analytical method, which was not sufficient to evaluate the levels of FAc in the aqueous humor and in the time points after month 6 for the retina in low dose group. In addition, the observed FAc levels in the retina and I/CB were notably more variable than for other ocular tissues. This is thought to be owing to variation in precision of the tissue dissection as small amounts of vitreous humor may contaminate the retina and I/CB samples because the vitreous humor is adherent to the retina, iris, and CB. In some dissections these attachments had to be cut using scissors. Due to the friable nature of the tissues, a rinsing step was not used. Although particles of other tissues contaminating the vitreous were removed and discarded and vitreous contamination of the retina and other tissues was removed when visible, cross-contamination cannot be ruled out. Finally, the study was terminated at month 24 in keeping with the ocular toxicity study. Early termination of sample prevented evaluation of the final elimination of FAc from ocular tissues. Despite these limitations, the study demonstrates sustained FAc delivery to ocular tissues over a 2-year period even with a single implant of the lowest dose, which is the marketed dose.

Subsequent to this study, it has been demonstrated that a single low dose implant provides measurable FAc in the aqueous humor of humans and is effective in the treatment of DME through month $36^{5,6}$ Thus, the FAc intravitreal implant has achieved long-term administration of low dose of a therapeutic agent to the intended ocular tissues without significant systemic exposure. 


\section{Acknowledgments}

The authors thank John Seng, PhD (formerly of Charles River Laboratories, Redfield, AR) for his support in the execution of the in life aspects of the study and Kevin Trimm, DESS (Charles River Laboratories Preclinical Services Montreal, Sennville, Quebec, Canada) for his input in the analysis of the pharmacokinetic data.

\section{Author Disclosure Statement}

F.E.K.: Alimera Sciences stockholder, consultant for Alimera Sciences, Inc. and other companies. K.E.G.: Employee and stockholder of Alimera Sciences, Inc. This study was sponsored by Alimera Sciences, Inc.

\section{References}

1. Jager, R.D., Aiello, L.P., Patel, S.C., and Cunningham, E.T. Risks of intravitreous injection: a comprehensive review. Retina. 24:676-698, 2004.

2. RETISERT ${ }^{\circledR}$ [Package Insert]. Bausch and Lomb, Inc., May 2012.

3. ILUVIEN ${ }^{\circledR}$ Summary of Product Characteristics. Alpharetta, GA: Alimera Sciences Limited; December 2013.

4. Driot, J.-Y., Novack, G.D., Rittenhouse, K.D., Milazzo, C., and Pearson, P.A. Ocular pharmacokinetics of fluocinolone acetonide after RETISERT ${ }^{\mathrm{TM}}$ Intravitreal Implantation in rabbits over a 1-year period. J. Ocular Pharm. Ther. 20:269275, 2004.

5. Campochiaro, P.A., Nguyen, Q.D., Hafiz, G., Bloom, S., Busquets, M., Ciulla, T., Feiner, L., Sabates, N., Billman, K., Kapik, B., Green, K., Kane, F.E., and FAMOUS Study Group. Aqueous levels of fluocinolone acetonide after administration of fluocinolone acetonide inserts or fluocinolone acetonide implants. Ophthalmology. 120:583-587, 2013.

6. Campochiaro, P.A., Brown, D.M., Pearson, A., Chen, S., Boyer, D., Ruiz-Moreno, J., Garretson, B., Gupta, A., Hariprasad, S.M., Bailey, C., Reichel, E., Soubrane, G., Kapik, B., Billman, K., Kane, F.E., Green, K., and for the FAME Study Group. Sustained delivery fluocinolone acetonide vitreous inserts provide benefit for at least 3 years in patients with diabetic macular edema, Ophthalmology. 119:21252132, 2012.

Received: September 3, 2014 Accepted: November 14, 2014

Address correspondence to: Dr. Kenneth E. Green Alimera Sciences, Inc. 6120 Windward Parkway

Suite 290

Alpharetta, GA 30005

E-mail: ken.green@alimerasciences.com 